\title{
A Note on Thomson's Characterizations of the Uniform Rule
}

\begin{abstract}
NIR DAGAN
Department of Economics, The Hebrew University of Jerusalem, 91905 Jerusalem, Israel

Received February 10, 1994; revised May 30, 1995

Thomson (Consistent solutions to the problem of fair division when preferences are single-peaked, J. Econ. Theory 63 (1994), 219-245) proved that the uniform rule of the fair division problem, where preferences are single-peaked, is the unique rule which is bilaterally consistent, continuous, Pareto optimal, and envy-free, in a setting of an infinite number of potential agents. We show that the uniqueness of the uniform rule is achieved without assuming continuity, even in a setting of a finite number of potential agents. A similar result is obtained by replacing envy-freeness with individual rationality from equal division. Journal of Economic Literature Classification Numbers: D63, C71. (c) 1996 Academic Press, Inc.
\end{abstract}

\section{InTRODUCTION AND SUMmary}

In this note we study the problem of fair division of an infinitely divisible and homogeneous commodity among agents that have single-peaked preferences. The most famous rule (single-valued solution concept) of this problem is the uniform rule.

The uniform rule satisfies some interesting properties. For example, it always assigns Pareto optimal and envy-free allocations. Clearly, in any given economy there are other allocations that satisfy these properties. Therefore, one may ask why the uniform rule is any better than any other Pareto optimal envy-free solution concept.

In a recent paper Thomson [5] gave interesting answers to this question. Thomson considered rules that satisfy various consistency properties. Assume that a rule recommends an allocation to a given economy; further assume that a set of agents wishes to reallocate its share of the commodity, and that this set of agents uses the same rule. Consistency requires that the allocation recommended to the agents of this smaller economy be identical to their part in the original one. Thomson [5] presented various axiomatic characterizations of the uniform rule using this consistency axiom or some of its variants. 
In this note we show that the impact of consistency is greater than implied by Thomson's [5, Theorems 1 and 2]. Thomson's Theorem 1 states that the uniform rule is the unique rule that satisfies bilateral consistency and continuity, and assigns Pareto optimal and envy-free allocations, in a setting of an infinite number of potential agents. We show that the uniqueness of the uniform rule is achieved without requiring continuity even in a setting of a finite number of potential agents. In our characterization, bilateral consistency is the only property that compares allocations of different economies. Thomson's Theorem 2 differs from the above in requiring the allocations to be individually rational from equal division instead of envy-free. This theorem too admits the same modification.

Thomson's proofs are based on his extensive study of multivalued solution concepts that are continuous and defined on the infinite-number-ofpotential-agents domain. Clearly, we cannot apply any of these results in proving our theorems. By restricting ourselves to a finite number of potential agents, we are not allowed to construct large economies in proving our results. Further, giving up continuity implies that we must use elementary arguments in our proofs. Indeed, the proofs use a simple argument to show that a rule that satisfies the axioms must be a function of the peaks only, for all two-agent economies. After that, completing the proofs is straightforward.

As our proofs use the "full power" of the single-valuedness of the rule, they cannot be applied in studying multivalued solutions. Moreover, Thomson brought to our attention that the result on multivalued solutions [Thomson, 5, Proposition 8] which he applied in his proof, does not hold for a domain with a finite number of potential agents.

Very recently, an extention of the fair division problem to economies with individual endowments was studied by Klaus et al. [1]. They established various characterizations of the uniform reallocation rule analogous to those obtained in the collective endowment case by modifying the consistency axiom and other axioms.

The model, the uniform rule, and some of its properties are presented in Section 2. The results are given in Section 3.

\section{The Model, the Axioms, and the Uniform Rule}

\subsection{The Model}

Definition. An economy is a pair $\left[\left(R_{i}\right)_{i \in Q}, M\right]$, where $M \in \mathbb{R}_{+}\left(\mathbb{R}_{+}\right.$is the set of nonnegative reals) and $Q$ is a finite set that contains at least two members. For all $i \in Q, R_{i}$ is a continuous, transitive, and complete preference relation on $\mathbb{R}_{+}$, which is single-peaked. That is, for all $i \in Q$, there exists a number $p\left(R_{i}\right)$ such that for all $x, y \in \mathbb{R}_{+}$if $y<x \leqslant p\left(R_{i}\right)$ or 
$p\left(R_{i}\right) \leqslant x<y$, then not $y R_{i} x$. We call $p\left(R_{i}\right)$ the peak of agent $i$, and denote $p\left(R_{i}\right)$ by $p_{i}$ and $\left(R_{i}\right)_{i \in Q}$ by $R_{Q}$.

Definition. An allocation in $\left(R_{Q}, M\right)$ is a list $\mathbf{x} \in \mathbb{R}_{+}^{Q}$ such that $\sum_{i \in Q} x_{i}=M$.

Definition. Let $\Omega$ be a non-empty set of economies. A rule on $\Omega$ is a function that assigns each economy in $\Omega$ a unique allocation.

\subsection{The Axioms}

Now we present some properties one may require of allocations and of rules. The first is equivalent to the usual condition of Pareto optimality.

Definition. An allocation $\mathbf{x}$ in $\left(R_{Q}, M\right)$ is Pareto optimal if and only if $\mathbf{x} \leqslant \mathbf{p}$ or $\mathbf{p} \leqslant \mathbf{x}$, where $\mathbf{p}=\left(p_{i}\right)_{i \in Q}$.

Definition. An allocation $\mathbf{x}$ in $\left(R_{Q}, M\right)$ is envy-free if for all $i, j \in Q$, $x_{i} R_{i} x_{j}$.

DEFINITION. An allocation $\mathbf{x}$ in $\left(R_{Q}, M\right)$ is individually rational from equal division if for all $i \in Q, x_{i} R_{i}(M / q)$, where $q$ is the number of agents in $Q$.

Definition. A rule $f$ on $\Omega$ is consistent if for all $\left(R_{Q}, M\right)$ in $\Omega$ :

$$
\begin{gathered}
{\left[f\left(R_{Q}, M\right)=\mathbf{x}, S \subset Q, \text { and }\left(R_{S}, \sum_{i \in S} x_{i}\right) \in \Omega\right]} \\
\Rightarrow f\left(R_{S}, \sum_{i \in S} x_{i}\right)=\mathbf{x} \mid S .
\end{gathered}
$$

A weaker property is bilateral consistency, which requires (1) only for subsets $S$ containing exactly two agents. We refer to $\left(R_{S}, \sum_{i \in S} x_{i}\right)$ as the reduced economy of $\left(R_{Q}, M\right)$ with respect to $S$ and $\mathbf{x}$.

Definition. A rule $f$ on $\Omega$ is conversely consistent if for all $\left(R_{Q}, M\right)$ : [ $\mathbf{x}$ is an allocation in $\left(R_{Q}, M\right)$, and for all $S \subset Q$ that contain exactly two agents, $\left(R_{S}, \sum_{i \in S} x_{i}\right) \in \Omega$ and $\left.\mathbf{x} \mid S=f\left(R_{S}, \sum_{i \in S} x_{i}\right)\right] \Rightarrow \mathbf{x}=f\left(R_{Q}, M\right)$.

\subsection{The Uniform Rule}

The uniform rule is defined as follows:

Definition. The uniform rule on $\Omega$ assigns each economy $\left(R_{Q}, M\right)$ in $\Omega$ the allocation $\mathbf{x}:=U\left(R_{Q}, M\right)$, where for all $i \in Q, x_{i}=\min \left(p_{i}, \lambda\right)$ if $M \leqslant \sum_{i \in Q} p_{i}$, and $x_{i}=\max \left(p_{i}, \lambda\right)$ if $M \geqslant \sum_{i \in Q} p_{i}$, where $\lambda$ solves the equation $\sum_{i \in Q} x_{i}=M$. 
The uniform rule satisfies all the properties presented above. Formally:

Lemma 1 (follows from Thomson [5, Propositions 4, 5, and 6]). The uniform rule assigns Pareto optimal, envy-free, and individually rational from equal division allocations, and it is consistent and conversely consistent.

\section{The Results}

Let $I$ be a set of natural numbers that contains at least two members. Denote by $\Omega^{I}$ the set of all economies $\left(R_{Q}, M\right)$ such that $Q \subset I$. Note that $Q$ must be finite by the definition of an economy. Domains of this structure are convenient for characterizing consistent rules, since any reduced economy of an economy in $\Omega^{I}$ is also in $\Omega^{I}$. Moreover, when the set of potential agents $I$ is infinite, consistency becomes more powerful. This is since, each economy in $\Omega^{I}$ is a reduced economy of other economies in $\Omega^{I}$. (Reduced economies are defined with the definition of consistency in Section 2.2). Examples of the use of this feature are Peleg's [3] and Lensberg's [2] characterizations of the core and the Nash bargaining solution, respectively.

Now we present a result by Thomson.

THEOREM 1 (Thomson [5, Theorem 1]). Let $\mathbb{N}$ be the set of natural numbers. The uniform rule is the unique rule on $\Omega^{\mathbb{N}}$ which is bilaterally consistent, continuous with respect to $M$, and assigns Pareto optimal and envyfree allocations.

And now the main result of this note:

THEOREM 2. Let I be a set of natural numbers that contains at least four members. The uniform rule is the unique rule on $\Omega^{I}$ which is bilaterally consistent and assigns Pareto optimal and envy-free allocations.

Proof. The proof follows from Lemma 1 above and Lemmata 2, 3, and 4 below.

Lemma 2. If a rule on $\Omega^{I}$ is bilaterally consistent and assigns Pareto optimal envy-free allocations, then for all two-agent economies it is a function of the peaks only; that is,

$\left[\left(R_{Q}, M\right),\left(R_{Q}^{\prime}, M\right) \in \Omega^{I}, \# Q=2, \forall i \in Q p_{i}=p_{i}^{\prime}\right] \Rightarrow f\left(R_{Q}, M\right)=f\left(R_{Q}^{\prime}, M\right)$. 
Proof. Let $\left(R_{Q}, M\right) \in \Omega^{I}, Q=\{i, j\}, i \neq j$. Let $S \subset I, S=\{k, l\}, k \neq l$, $S \cap Q=\varnothing$. Consider the four-agent economy $\left(R_{Q \cup S}, 2 M\right)$, where $p_{i}=p_{k}$ and $p_{j}=p_{l}$. Let $\mathbf{x}=f\left(R_{Q \cup S}, 2 M\right)$. By Pareto optimality $\mathbf{p} \leqslant \mathbf{x}$ or $\mathbf{p} \geqslant \mathbf{x}$, this together with envy-freeness implies $x_{i}=x_{k}$ and $x_{j}=x_{l}$. Thus we have $x_{i}+x_{j}=x_{k}+x_{l}=M$. Applying bilateral consistency with respect to $S$ and with respect to $Q$ we get $f_{i}\left(R_{Q}, M\right)=x_{i}=x_{k}=f_{k}\left(R_{S}, M\right)$ and $f_{j}\left(R_{Q}, M\right)=x_{j}=x_{l}=f_{l}\left(R_{S}, M\right)$. Now, let $R_{i}^{\prime}$ and $R_{j}^{\prime}$ be preference relations with $p_{i}=p_{i}^{\prime}$ and $p_{j}=p_{j}^{\prime}$. By applying an identical argument as above to the economy $\left(R_{Q \cup S}^{\prime}, 2 M\right)$, where $R_{i}^{\prime}=R_{k}^{\prime}$ and $R_{j}^{\prime}=R_{l}^{\prime}$, the lemma is proved.

Lemma 3 (follows from Thomson [4, Lemma 1]). If a rule $f$ on $\Omega^{I}$ is a function of the peaks only for all two agent economies, and it assigns Pareto optimal and envy-free allocations, then it coincides with the uniform rule for all two agent economies.

The proof is given for completeness, as the lemma is already known.

Proof. First, consider a two agent economy $\left(R_{Q}, M\right) \in \Omega^{I}$, with $Q=\{i, j\}, i \neq j$, and $p_{i}+p_{j} \geqslant M$. Let $\mathbf{x}=f\left(R_{Q}, M\right)$. By Pareto optimality $\mathbf{x} \leqslant \mathbf{p}$. Assume without loss of generality that $p_{i} \leqslant p_{j}$. Now, if $x_{j} \leqslant p_{i}$, envyfree implies $x_{i}=x_{j}$. If $x_{j}>p_{i}$, we claim that it must be that $x_{i}=p_{i}$. Assume not, then $x_{i}<p_{i}$ and $x_{j}>p_{i}$. It is easy to find a preference relation $R_{i}^{\prime}$ with $p_{i}^{\prime}=p_{i}$ and not $x_{i} R_{i} x_{j}$. Since $f$ is a function of the peaks only, $f\left[\left(R_{i}^{\prime}, R_{j}\right), M\right]=\mathbf{x}$, which violates envy-free for agent $i$. An analogous argument can be applied to show the required result for the case where $p_{i}+p_{j}<M$.

Lemma 4. If a rule $f$ on $\Omega^{I}$ is bilaterally consistent and coincides with the uniform rule for all two agent economies, then $f$ is the uniform rule.

Proof. Let $\left(R_{Q}, M\right) \in \Omega^{I}$, and let $\mathbf{x}=f\left(R_{Q}, M\right)$. By bilateral consistency and since $f$ coincides with the uniform rule for all two agent economies, we have that for all $S \subset Q$ with $\# S=2, \mathbf{x} \mid S=f\left(R_{S}, \sum_{i \in S} x_{i}\right)=U\left(R_{S}, \sum_{i \in S} x_{i}\right)$. Now, since the uniform rule is conversely consistent, $\mathbf{x}=U\left(R_{Q}, M\right)$.

Thomson [5, Theorem 2] states that envy-freeness may be replaced with individual rationality from equal division in Theorem 1 . This replacement is possible also here, thus continuity and infinite number potential agents are not needed in this characterization as well. Formally we have:

THEOREM 3. Let I be a set of natural numbers that contains at least four members. The uniform rule is the unique rule on $\Omega^{I}$ which is bilaterally consistent and assigns Pareto optimal and individually rational from equal division allocations. 
Proof. The proof follows from Lemmata 1 and 4 above, and 5 and 6 below.

Lemma 5. If a rule on $\Omega^{I}$ is bilaterally consistent and assigns Pareto optimal and individually rational from equal division allocations, then for all two-agent economies it is a function of the peaks only (see definition in Lemma 2.)

Proof. Let $\left(R_{Q}, M\right) \in \Omega^{I}, Q=\{i, j\}, i \neq j$. Let $S \subset I, S=\{k, l\}, k \neq l$, $S \cap Q=\varnothing$. Consider, as in Lemma 2, the four-agent economy $\left(R_{Q \cup S}, 2 M\right)$, where $p_{i}=p_{k}$ and $p_{j}=p_{l}$. Let $\mathbf{x}=f\left(R_{Q \cup S}, 2 M\right)$. Now consider the reduced economy with respect to $\{i, k\}$. This reduced economy has a unique Pareto optimal and individually rational from equal division allocation in which $x_{i}=x_{k}$. Similarly, the same holds for $j$ and $l$. The rest of the proof is identical to the proof of Lemma 2.

Lemma 6. If a rule $f$ on $\Omega^{I}$ is a function of the peaks only for all two agent economies, and it assigns Pareto optimal and individually rational from equal division allocations, then it coincides with the uniform rule for all twoagent economies.

Proof. First, consider a two-agent economy $\left(R_{Q}, M\right) \in \Omega^{I}$, with $Q=\{i, j\}, i \neq j$, and $p_{i}+p_{j} \geqslant M$. Let $\mathbf{x}=f\left(R_{Q}, M\right)$. Assume without loss of generality that $p_{i} \leqslant p_{j}$. Now, if $M \leqslant 2 p_{i}$, individual rationality from equal division implies $x_{i} \geqslant M / 2$ and $x_{j} \geqslant M / 2$; thus $x_{i}=x_{j}$. If $M>2 p_{i}$, we claim that it must be that $x_{i}=p_{i}$. Assume not; then by Pareto optimality $x_{i}<p_{i}$ and $M / 2>p_{i}$. It is easy to find a preference relation $R_{i}^{\prime}$ with $p_{i}^{\prime}=p_{i}$ and not $x_{i} R_{i}(M / 2)$. Since $f$ is a function of the peaks only, $f\left[\left(R_{i}^{\prime}, R_{j}\right), M\right]=\mathbf{x}$, which violates individual rationality from equal division for agent $i$. Now, consider the case where $p_{i}+p_{j}<M$. Assume without loss of generality that $p_{i} \leqslant p_{j}$. Now, if $M \geqslant 2 p_{j}$, individual rationality from equal division implies that $x_{i} \leqslant M / 2$ and $x_{j} \leqslant M / 2$, thus $x_{i}=x_{j}$. If $M<2 p_{j}$, we claim that it must be that $x_{j}=p_{j}$. Assume not; then by Pareto optimality $x_{j}>p_{j}$ and $M / 2<p_{j}$. It is easy to find a preference relation $R_{j}^{\prime}$ with $p_{j}^{\prime}=p_{j}$ and not $x_{i} R_{j}(M / 2)$. Since $f$ is a function of the peaks only, $f\left[\left(R_{i}, R_{j}^{\prime}\right), M\right]=\mathbf{x}$, which violates individual rationality from equal division for agent $j$.

\section{ACKNOWLEDGMENTS}

This note is based on Chapter 3 of the author's Ph.D. thesis. The author gratefully acknowledges important comments from Sergiu Hart, William Thomson, and Eyal Winter. 


\section{REFERENCES}

1. B. Klaus, H. Peters, and T. Storcken, Reallocation of a perfectly divisible good, mimeo, Maastricht, 1995.

2. T. LensBerg, Stability and the Nash solution, J. Econ. Theory 45 (1988), 330-341.

3. B. Peleg, An axiomatization of the core of cooperative games without side payments, J. Math. Econ. 14 (1985), 203-214.

4. W. Thomson, Resource-monotonic solutions to the problem of fair division when preferences are single-peaked, Soc. Choice Welfare 10 (1993), 1-19.

5. W. Thomson, Consistent solutions to the problem of fair division when preferences are single-peaked, J. Econ. Theory 63 (1994), 219-245. 